\title{
Vacancy formation in homoepitaxially grown Ag films and its effect on surface morphology
}

\author{
C. E. Botez, W. C. Elliott, and P. F. Miceli \\ Department of Physics and Astronomy, University of Missouri-Columbia, Columbia, Missouri 65211 \\ P. W. Stephens \\ Department of Physics, State University of New York, Stony Brook, New York 11794 \\ (Received 20 December 2001; revised manuscript received 27 March 2002; published 26 August 2002)
}

\begin{abstract}
Synchrotron x-ray diffraction was used to investigate the low-temperature homoepitaxial growth on $\operatorname{Ag}(001)$ and $\mathrm{Ag}(111)$ surfaces. For both orientations, the $\mathrm{Ag}$ films deposited at $T=100 \mathrm{~K}$ were observed to exhibit a $1 \%$ surface-normal compressive strain, indicating that an appreciable vacancy concentration $(\sim 2 \%)$ is incorporated in the growing film. Concomitantly with the incorporation of vacancies, the growth on $\mathrm{Ag}(111)$ leads to the formation of pyramidlike structures with a non-Gaussian distribution of heights, whereas a similar effect was not observed for $\operatorname{Ag}(001)$.
\end{abstract}

DOI: 10.1103/PhysRevB.66.075418

PACS number(s): $61.10 . \mathrm{Kw}, 68.55 . \mathrm{Jk}$

\section{INTRODUCTION}

In several studies of metal homoepitaxy, ${ }^{1,2}$ the surface roughness of the deposited film was observed to decrease when the growth was carried out at progressively lower temperatures. This inverse temperature dependence of roughening [or "reentrant smooth growth" (RSG)] is somewhat surprising, since the lowering of $T$ inhibits the adatom diffusion, which is the main smoothening mechanism in epitaxial growth. Motivated by the observation of a RSG for the $\mathrm{Cu}(001)$ (Ref. 1) and Pt(111) (Ref. 2) homoepitaxy, a model was proposed where the lowering of the temperature below a certain value enables an additional smoothening process that causes the atoms deposited at step edges to "funnel" down to lower fourfold hollow absorption sites. ${ }^{3}$ This "downward funneling," which was quite successful ${ }^{4,5}$ in explaining the reentrant smooth growth on (001) surfaces, is believed to be continuously enhanced by the reduction of the temperature and, therefore, one should expect that the growth becomes progressively smoother as $T$ is decreased toward $0 \mathrm{~K}$. More recently, however, scanning tunneling microscopy (STM) measurements of $\mathrm{Ag} / \mathrm{Ag}(001)$ epitaxy $^{6}$ have indicated that, indeed, the roughness of the deposited films decreases when $T$ is lowered from 200 to $130 \mathrm{~K}$, but surprisingly, it increases upon further cooling to $50 \mathrm{~K}$. To explain this behavior, without discarding the downward funneling hypothesis, the authors of Ref. 6 propose a scenario where some of the depositing atoms get trapped on the sides of nanoprotrusions (which are more numerous at low $T$ ) instead of funneling down over the step edges. Molecular-dynamics simulations of low-temperature growth ${ }^{7}$ suggest that this "restricted downward funneling" can lead to the formation and incorporation of internal voids in the growing film. While such a behavior is plausible at very low temperatures, direct experimental evidence for a large number of vacancies incorporated in a homoepitaxially grown film is not yet available. Such a finding would be of particular importance because it imposes further limitations on most of the homoepitaxial growth models, which assume that, regardless of the temperature, the deposited atoms occupy a perfect crystal lattice identical to that of the underlying substrate. Moreover, the potential presence of vacancies in homoepitaxially grown metal films raises several interesting questions: What is the actual concentration of the incorporated vacancies and how does it change with the temperature? Is this vacancy formation typical for the (001) surfaces (which are more likely to exhibit downward funneling) or can it also occur for other orientations? How does the vacancy formation influence the morphology of the evolving surface?

The purpose of the present work is to investigate the possible presence of a large vacancy concentration in a homoepitaxial film grown at low temperatures and to address some of the questions above. To accomplish this goal, we have studied the growth of $\mathrm{Ag}$ on $\operatorname{Ag}(001)$ and $\operatorname{Ag}(111)$, at $T=100 \mathrm{~K}$, using synchrotron $\mathrm{x}$-ray diffraction (XRD). The choice of XRD as a probe is quite natural given the fact that, having a large penetration length, $x$ rays are highly sensitive to any defects below the surface that introduce structural differences between the deposited film and the underlying bulk crystal. Furthermore, synchrotron XRD is well established as a tool that can reveal details of the surface structure on an atomic scale ${ }^{8}$ and it has been successfully used in studies of surface crystallography, ${ }^{9}$ surface morphology, ${ }^{10}$ and surface thermal expansion. ${ }^{11}$ By using this method in our present experiments we have the ability to simultaneously measure the evolving surface morphology and to test if any defects are incorporated in the growing film. Our specular reflectivity data show that a substantial compressive strain is present in the $\mathrm{Ag}$ films deposited at $T=100 \mathrm{~K}$ on $\mathrm{Ag}(001)$ and $\operatorname{Ag}(111)$ substrates, indicating that a large number of vacancies are incorporated in the growing film. From the magnitude of the strain we estimate that the vacancy concentration is approximately $2 \%$ for both orientations. The vacancies anneal at room temperature. We also find that the growth on $\mathrm{Ag}(111)$, in the presence of vacancies, gives rise to a surface morphology having terraces with a non-Gausian distribution of heights. Interestingly, such an effect is not present for the growth on $\operatorname{Ag}(001)$.

\section{EXPERIMENTAL PROCEDURE}

Our experiments were performed on the SUNY X3B2 beamline at the National Synchrotron Light Source, 
Brookhaven National Laboratory, using a custom-designed ultrahigh-vacuum (UHV) diffractometer. The UHV chamber was equipped with an ion pump as well as a titanium sublimation pump, allowing the base pressure to reach $10^{-10}$ Torr. The $\operatorname{Ag}(001)$ and $\operatorname{Ag}(111)$ samples were mechanically polished to reduce the miscut to about $0.1^{\circ}$ and subsequently prepared in UHV by repeated cycles of $15-\mathrm{min}$ $\mathrm{Ar}^{+}$sputtering $\left(p=10^{-5}\right.$ Torr, acceleration voltage $=1 \mathrm{kV}$, and sputtering current $=10 \mu \mathrm{A})$ and 1-h high-temperature annealing at $T=1000 \mathrm{~K}$ (achieved by electron bombardment). As a result of this treatment, we routinely obtained high-quality starting surfaces that are virtually flat at an atomic level (rms roughness $<0.5 \AA$ ), have a significantly reduced mosaic spread $\Delta \omega_{0}$, and have a very large averagesize-terrace diameter (in-plane correlation length) $L_{\text {ave }}$. For example, room-temperature measurements of the as-prepared $\operatorname{Ag}(111)$ surface yield $\Delta \omega_{0}=0.04^{\circ}$ and $L_{\text {ave }}=8000 \AA$. Here $\mathrm{Ag}$ was evaporated from a resistively heated crucible and deposited onto the surface of the sample at a rate measured by a calibrated quartz monitor. For $\operatorname{Ag}(001)$, a more precise measurement of the deposition rate was achieved by following the intensity oscillations of the out-of-phase specular reflection. A rate of $1 \mathrm{ML} / \mathrm{min}$ was chosen for both $\operatorname{Ag}(001)$ and $\operatorname{Ag}(111)$ homoepitaxy. Liquid nitrogen was used to cool the sample during deposition, while the temperature was monitored and stabilized $( \pm 1 \mathrm{~K})$ by a temperature controller. Particular attention was paid to the cleanliness of the surface where Auger electron spectroscopy (AES) was used to detect impurities. AES measured for the cooled substrates as well as subsequently grown films consistently showed no trace of impurities, even when the samples were allowed to sit for more than an hour. X-ray scattering data were collected at $T=100 \mathrm{~K}$, from both $\operatorname{Ag}(001)(\lambda=1.0207 \AA)$ and $\operatorname{Ag}(111)$ $(\lambda=1.1379 \AA)$, by scanning across the specular rod ("rocking" scans) for an extended range of values of the surfacenormal scattering wave vector $Q_{z}$. At each $Q_{z}$, the specular reflectivity was obtained from the corresponding rocking scan. ${ }^{8}$

\section{RESULTS AND DISCUSSION}

Figure 1 shows the specular reflectivity, measured around the (111) Bragg reflection, from the $\operatorname{Ag}(111)$ surface with 5 ML deposited at (a) $200 \mathrm{~K}$ and (b) $100 \mathrm{~K}$ (open symbols). As can be observed, lowering the temperature has a dramatic effect on the reflectivity. At $200 \mathrm{~K}$, the line shape is almost symmetric and can be excellently described by a simple model $^{12}$ (dashed curve), which assumes that the surface roughness, consisting of a Poisson distribution of surface heights, is the only deviation from a perfectly truncated crystal. Such an analysis works very well at temperatures above $200 \mathrm{~K}$, as demonstrated in our previous kinetic roughening studies of $\mathrm{Ag} / \mathrm{Ag}(001)$ and $\mathrm{Ag} / \mathrm{Ag}(111) .{ }^{13}$ When the film is grown at $T=100 \mathrm{~K}$, however, there are essentially two significant changes in the reflectivity line shape [Fig. 1(b)]. First, we observe thin-film interference fringes, which suggest that there are structural differences between the deposited film and the underlying bulk crystal substrate. The fringe spacing changes with the film thickness, and this is demon-

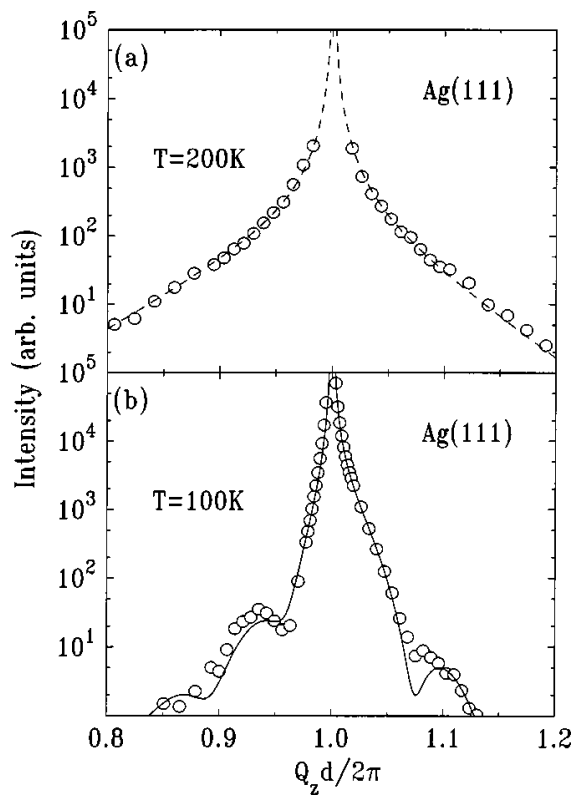

FIG. 1. Specular reflectivity from the $\operatorname{Ag}(111)$ surface, with 5 ML deposited at (a) $T=200 \mathrm{~K}$ and (b) $T=100 \mathrm{~K}$ (open symbols). At $200 \mathrm{~K}$, the data are well described by a simple Gaussian surface roughness (Ref. 12) (dashed line) while at $100 \mathrm{~K}$ a real-space model that includes a large compressive strain in the deposited film is necessary to fit (solid line) the interference fringes and the pronounced asymmetry toward higher perpendicular wave vector.

strated by "real-time" measurements, shown in Fig. 2, for two fixed grazing-incidence detector positions $\left(2 \theta=2.0^{\circ}\right.$ and $2 \theta=1.5^{\circ}$ ) where the fringes can be seen "rolling" by the detector as the film thickness increases. The second feature in Fig. 1(b) is that a pronounced asymmetry towards higher $Q_{z}$ develops around the (111) Bragg reflection, indicating the presence of a compressive strain. This second observation, in fact, explains the first: the slight difference in lattice parameter provides "contrast" between the film and substrate, thereby leading to interference fringes around the Bragg reflection. Both features of the x-ray line shape are found to vanish upon subsequent warming of the sample to $300 \mathrm{~K}$.

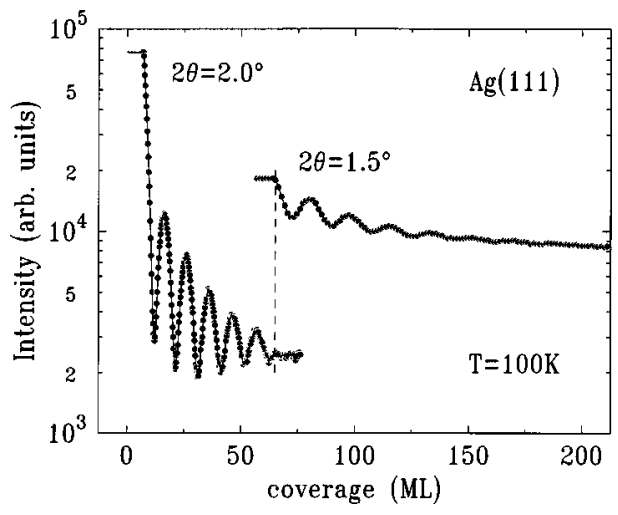

FIG. 2. Intensity measured at two fixed grazing-incidence detector positions during deposition on $\mathrm{Ag}(111)$ surface exhibits interference fringes, suggesting that the homoepitaxially grown film differs from the underlying bulk substrate. 

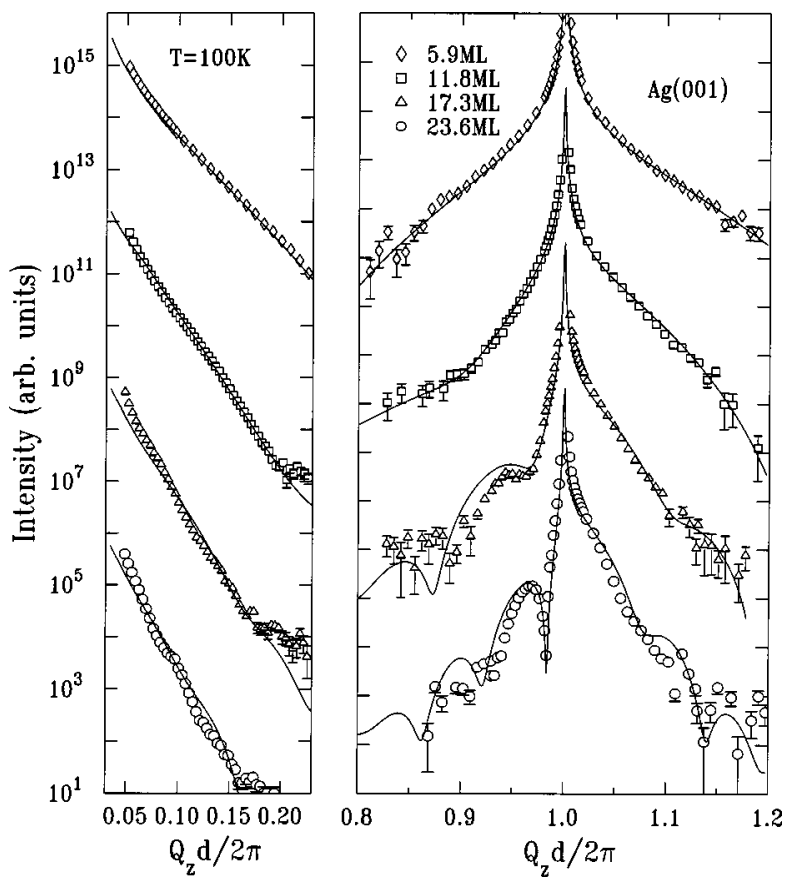

FIG. 3. Specular reflectivity measured for $\operatorname{Ag}(001)$ with $5.9 \mathrm{ML}$ (diamonds), 11.8 ML (squares), 17.3 ML (triangles), and 23.6 ML (circles), deposited at $T=100 \mathrm{~K}$. The curves are vertically shifted for clarity. The solid lines represent best fits to a real-space model where a compressive strain is assumed to be present in the deposited film.

Figures 3 and 4 show the specular reflectivity data obtained from $\mathrm{Ag} / \mathrm{Ag}(001)$ and $\mathrm{Ag} / \mathrm{Ag}(111)$, respectively. The data were collected over an extended range of surfacenormal wave vector $Q_{z}$ at $T=100 \mathrm{~K}$ and four different coverages $\Theta$. Comparing the two sets of data, we observe the same features (interference fringes and asymmetry) around the Bragg reflection for both $\operatorname{Ag}(001)$ and $\operatorname{Ag}(111)$, indicating a similar behavior in terms of the strain. Our analysis, discussed in detail below, shows that films grown at $100 \mathrm{~K}$ on both orientations generally exhibit a $\sim 1 \%$ surface-normal compressive strain or $\left(d_{\text {film }}-d_{\text {subst }}\right) / d_{\text {subst }}=-1 \%$, where $d_{\text {film }}$ is the interplanar spacing of the film along the surface normal and $d_{\text {subst }}$ is the interplanar spacing of the substrate. An important difference between these data sets, however, occurs at low values of $Q_{z}$ where interference fringes are observed for the $\operatorname{Ag}(111)$ homoepitaxy, but are absent for $\mathrm{Ag}(001)$. It will be shown that the surface morphology of $\mathrm{Ag}(111)$ is responsible for the appearance of these fringes and that strain alone does not give interference fringes at low $Q_{z}$.

Beginning with the analysis of the reflectivity from the $\mathrm{Ag}(001)$ surface (Fig. 3), we consider the simplest model where, in addition to some surface roughness, ${ }^{13}$ the film has a lattice parameter different from that of the substrate. As can be seen, such a model provides a good description of the data: the least-squares fits (solid curves in Fig. 3) match both the interference fringes and the asymmetry about the Bragg reflection as well as the absence of interference fringes at low $Q_{z}$. At all coverages the best fits yield ap-
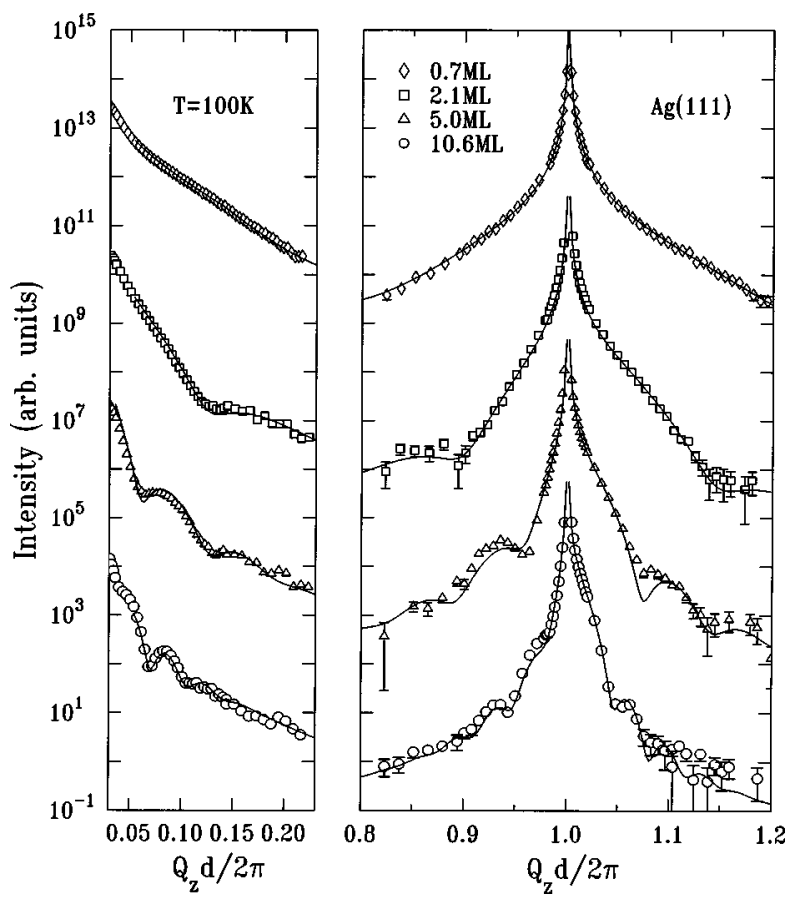

FIG. 4. Specular reflectivity measured for $\mathrm{Ag}(111)$ with $0.7 \mathrm{ML}$ (diamonds), 2.1 ML (squares), 5.0 ML (triangles), and 10.6 ML (circles) thick films, deposited on $\mathrm{Ag}(111)$ at $T=100 \mathrm{~K}$. The curves are vertically shifted for clarity. The best fits (solid lines) are based on a model where, in addition to the surface-normal strain in the deposited films, a surface morphology consisting of pyramidlike structures is assumed.

proximately the same value of $\sim 1 \%$ for the magnitude of the compressive strain.

Now we discuss the origin of the observed strain. First, we underscore that the strain cannot arise from an accidental low-temperature surface contamination because AES, measured before and after deposition, consistently showed clean surfaces with an AES sensitivity limit lower than the large impurity concentration that would be necessary to account for the observed strain magnitude. Degrading the vacuum did not change our low-temperature $\mathrm{x}$-ray scattering or AES results-this is a manifestation of the low reactivity of $\mathrm{Ag}$ surfaces. Molecular hydrogen, which is not detectable by AES, has a very low sticking probability at $100 \mathrm{~K}$ (Ref. 14) and there is no source of atomic hydrogen. Furthermore, the presence of interstitial impurities or substitutional impurities larger than Ag would cause a lattice expansion rather than the contraction found in our experiment. We can also eliminate the hypothesis that stacking faults create the strain. Indeed, such defects can occur in (111) homoepitaxy. However, we observe a similar compressive strain for films grown on both the (111) and (001) surfaces, where the latter cannot have stacking faults. Moreover, $\mathrm{x}$-ray measurements that reveal stacking faults on homoepitaxially grown $\mathrm{Ag}(111)$ (Ref. 15) and $\mathrm{Cu}(111)$ (Ref. 16) do not exhibit a compressive strain and our measurements find no strain at the temperatures where the stacking faults were observed. It should also be noted that grain boundaries, which could become more populous at low temperature, would lead to a "mosaic crystal," ${ }^{17}$ but would not shift the Bragg position of the film. 


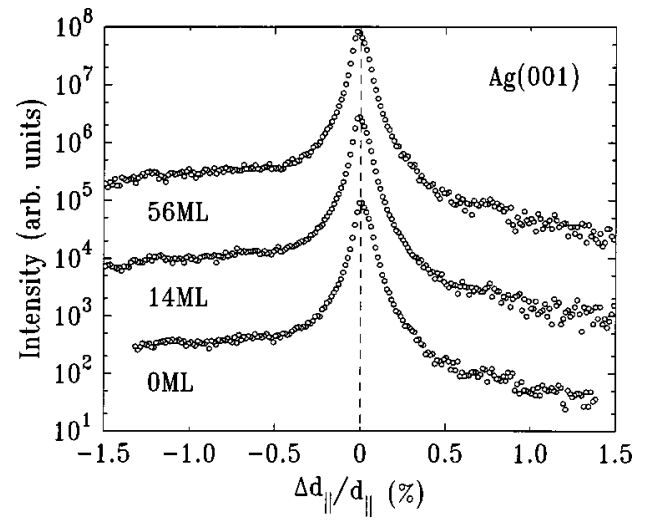

FIG. 5. In-plane (020) Bragg reflection was measured with the wave vector parallel to the surface for different coverages and shows no evidence of lateral strain in the film deposited at low temperature on $\operatorname{Ag}(001)$. The incident and outgoing beams were kept below the critical angle to enhance the surface sensitivity.

We do not see evidence of conventional mosaicity in the deposited films, but rather observe a coherent crystalline film on top of the substrate. With these points in mind, several factors lead us to conclude that the compressive strain observed in our experiments arises from the incorporation of vacancies in the film, which is grown under far-fromequilibrium conditions. First, it should be noted that the large negative volume of a vacancy is necessary in order to explain the relatively large compressive strain. Second, the observed annealing of the line shape by warming to $300 \mathrm{~K}$ is consistent with vacancies: radiation damage studies of bulk Ag show that vacancies anneal at $260 \mathrm{~K} .{ }^{18}$ Finally, computer simulations of low- $T$ metal homoepitaxy have predicted that vacancies or internal voids (vacancy clusters) can be incorporated into a growing film. ${ }^{7}$

The concentration of vacancies can be estimated from the measured strain. To do that, we first check if the lattice distortion occurs only in the surface-normal direction (obtained from the specular reflectivity line shape) or if there is also strain in the plane of the surface. Figure 5 shows diffraction measurements taken across the (020) in-plane Bragg reflection to look for lateral strain in $\mathrm{Ag}$ films of different thickness deposited on $\operatorname{Ag}(001)$ at low temperature. To enhance the surface sensitivity (to $\sim 50 \AA$ ), both the incident and outgoing beams were set at grazing angles below the critical angle for total reflection. A change in the in-plane lattice parameter of the film is expected to cause a second displaced diffraction peak or, at least, a pronounced elongation of the main diffraction peak. ${ }^{19}$ That such features are not observed in Fig. 5, where the horizontal axis is given directly in terms of $\Delta d_{\|} / d_{\|}$, leads to the conclusion that the in-plane lattice parameter of the film is identical to that of the substrate. Such pseudomorphic growth with uniaxial strain is commonly observed in heteroepitaxial systems. ${ }^{20}$ Using a Vegard's law (linear) relationship ${ }^{21}$ between the concentration of point defects and the defect volume, which is then corrected for the uniaxial strain, ${ }^{22}$ we obtain

$$
\frac{d_{\text {film }}-d_{\text {subst }}}{d_{\text {film }}}=-\alpha\left(1+\frac{2 C_{12}}{C_{11}}\right) c_{v},
$$

where $c_{v}$ is the vacancy concentration, $\alpha=0.2$ relates the strain to the vacancy concentration in the bulk metal, ${ }^{23}$ and $C_{12} / C_{11}=0.76$ is the ratio of elastic constants that accounts for the lateral elastic clamping of the film. Using our measured strain of $\sim 1 \%$, we find $c_{v} \approx 2 \%$ for the concentration of vacancies in the deposited films. Returning to the $\mathrm{Ag} /$ $\operatorname{Ag}(001)$ reflectivity data in Fig. 3, we can now say that the absence of interference fringes at low angles is due to the fact that the scattering power of the film is almost identical to that of the substrate. Indeed, the $1 \%$ change in the lattice parameter of the film and the associated $2 \%$ concentration of vacancies are much too small to induce significant density differences between the film and underlying bulk crystal. On the other hand, the strained film is distinguishable from the substrate in the vicinity of the Bragg reflection, where the wave vector is comparable to (and therefore can probe) the interatomic distances.

The coverage-dependent data for $\operatorname{Ag}(111)$ (Fig. 4) show the same strain-induced features around the Bragg reflection, but in addition, strong interference fringes appear in the low$Q_{z}$ region. Given the evolution of their periodicity with the coverage, it is obvious that these fringes are related to the film thickness. Yet since the magnitude of the strain in the $\mathrm{Ag} / \mathrm{Ag}(111)$ films is similar to that of $\mathrm{Ag} / \mathrm{Ag}(001)$, the fringes cannot be directly ascribed to a change in density via the vacancies. Instead, we will show that the effect comes from the surface morphology that develops concomitantly with the vacancy formation.

It is well known that the homoepitaxial growth on certain metallic surfaces leads to three-dimensional pyramidlike mound structures with a characteristic lateral separation and selected slope. ${ }^{24}$ This type of growth arises from instabilities created by an additional energy barrier to diffusion over crystalline step edges - the so-called Ehrlich-Schwoebel (ES) barrier. ${ }^{25}$ Experiments to determine the root-mean-square roughness of a growing surface are usually well characterized in terms of a binomial distribution (e.g., Gaussian, Poisson) of surface heights. However, there is no reason to expect, a priori, that these surfaces, which possess pyramidlike structures, should always lead to such a distribution. Indeed, even the effect of a smooth starting surface could cause asymmetric height fluctuations, leading to a non-Gaussian distribution: for small asymmetry, a perturbation expansion $^{4}$ about a Gaussian-distributed surface gives the "skewness" as the leading-order correction, and this has been observed in STM experiments. ${ }^{4}$ It should be noted that $\operatorname{Ag}(111)$ has a rather large ES barrier ${ }^{26}$ where large mounds develop during homoepitaxial growth, and these mounds are anticipated to be most pronounced at low temperatures. Therefore, we analyzed the $\operatorname{Ag}(111)$ reflectivity data using a model that, in addition to strain in the deposited film, includes pyramidal surface structures.

Since specular reflectivity gives information on the vertical surface height, we consider the exposed surface fraction $P_{j}$ at a crystalline layer height $j$, where $j=0$ is taken as the substrate surface layer. Three-dimensional pyramids are incorporated into a simple model for the reflectivity by recognizing that the exposed surface fraction changes linearly with $j$ and, superimposed on this, the pyramid heights will fluctu- 


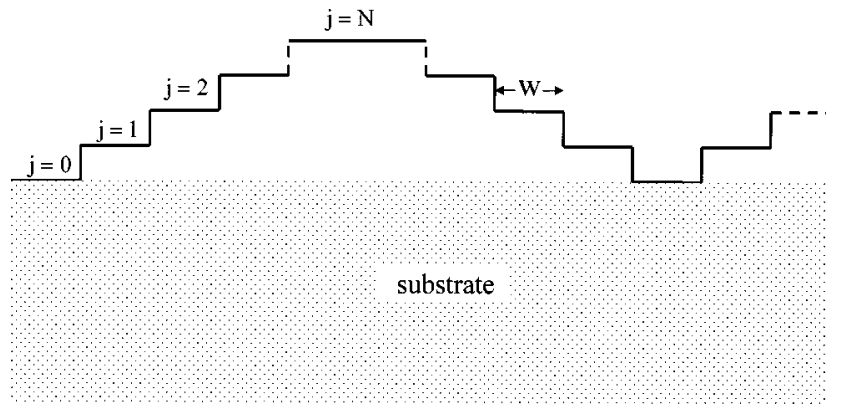

FIG. 6. Schematic diagram showing a cross section through a typical pyramid. Each terrace is assumed to have the same width $W$, which leads to an exposed area that decreases linearly with increasing terrace level $j$.

ate. The basic parameters of the model are indicated in the diagram of Fig. 6, which shows a cross section through a typical pyramid. For a single flat-topped pyramid having $N$ layers with equal terrace widths $W$ and an exposed surface fraction of the top layer, $\theta_{\text {top }}$, we calculate the layerdependent exposed surface fraction

$$
\begin{gathered}
P_{j}^{\mathrm{pyr}}=b-a j, \quad j \leqslant N-1, \\
P_{j}^{\mathrm{pyr}}=\theta_{\mathrm{top}}, \quad j=N,
\end{gathered}
$$

where $a=2 \alpha^{2}, b=2 \alpha-\alpha^{2}$, and $\alpha=\left(1-\sqrt{\theta_{\text {top }}}\right) / N$ are obtained by requiring normalization of the surface fraction, $\sum_{j=0}^{N} \mathbf{P}_{j}^{\mathrm{pyr}}=1$. Here $\alpha=2 W / D$ relates the terrace width $W$ to the mound separation $D$, but neither $W$ nor $D$ can be determined independently, since specular reflectivity does not provide information on the lateral length scale. Because not all pyramids have the same height, we assume fluctuations having a binomial distribution of the pyramid heights, $P_{j}$ $=\left\langle P_{j}^{\mathrm{pyr}}\right\rangle_{N}$, which defines an average pyramid height $N_{\text {pyr }}$ and pyramid height variance $S_{\text {pyr }}^{2}$. The specular reflectivity is then calculated ${ }^{27}$ from the film and substrate contributions:

$$
R \propto \frac{\left|f\left(Q_{z}\right)\right|^{2}}{Q_{z}^{2}}\left|V_{0}\right|^{2}\left|\frac{1}{1-e^{-i Q_{2} d_{\text {subst }}}}+\left(1-c_{v}\right) \frac{V_{p} V_{b}-1}{1-e^{-i Q_{z} d_{\text {film }}}}\right|^{2},
$$

where $f\left(Q_{z}\right)$ is the atomic form factor of $\mathrm{Ag}$ and $c_{v}$, determined through Eq. (1), makes a small correction for the vacancy-induced change in film density. $V_{0}$ accounts for the roughness of the starting substrate surface, which is negligible, so we take $V_{0}=1$. Here $V_{b}$ allows for a fluctuation in the position of the base of the pyramids, but this was also found to be negligible $\left(V_{b}=1\right)$ during the analysis of the data. $V_{p}$ incorporates the effects of the pyramidal surface morphology and is given by

$$
V_{p}=\left(1-\theta_{b}\right)+\theta_{b} \sum_{j} P_{j} e^{i Q_{z} d_{\mathrm{film}} j}
$$

where $\theta_{b}$ is introduced as a shape correction and is effectively the fraction of the surface covered by pyramids. Although the model is a highly simplified description of the surface, the linear dependence of $P_{j}^{\mathrm{pyr}}$ on $j$ captures the es-
TABLE I. Parameters determined from the fits to the $\operatorname{Ag}(111)$ data in Fig. 4.

\begin{tabular}{ccccccc}
\hline \hline$\Theta^{\text {expt }}$ & $\Theta^{\text {comp }}$ & $\sigma$ & $N_{\text {pyr }}$ & $S_{\text {pyr }}$ & $\theta_{\text {top }}$ & $\theta_{b}$ \\
\hline 0.7 & 1.32 & 1.31 & 2.10 & 1.25 & 0.50 & 1.0 \\
2.1 & 3.34 & 2.49 & 5.45 & 1.87 & 0.38 & 1.0 \\
5.0 & 4.50 & 4.91 & 12.63 & 1.94 & 0.23 & 0.9 \\
10.6 & 11.80 & 8.90 & 25.20 & 2.75 & 0.14 & 0.9 \\
\hline \hline
\end{tabular}

sential feature that the exposed area varies with layer height. The most significant corrections to this pyramid shape will occur towards the top and bottom where there can be a substantial increase in the exposed area-this is approximated by $\theta_{\text {top }}$ and $\theta_{b}$ where the corrections are implemented in a single layer at the top and bottom of the pyramid, respectively. Allowed to vary in the fit were an overall intensity scale factor, $N_{\text {pyr }}, S_{\text {pyr }}, \theta_{\text {top }}$, and $d_{\text {film }}$. Note that these parameters are quite uncorrelated among each other: $N_{\text {pyr }}$ determines the oscillation period at low angle, and $d_{\text {film }}$ determines the asymmetry around the Bragg reflection, whereas $\theta_{\text {top }}$ and $S_{\text {pyr }}$ affect the magnitude and decay of the oscillations. It was found that the fits required $\theta_{b}$ to be near unity, but were otherwise insensitive to the actual value: thus, $\theta_{b}$ was fixed within the range 0.95 \pm 0.05 .

The best fits to the $\operatorname{Ag}(111)$ reflectivity data, represented by the solid curves in Fig. 4, match the asymmetries around the Bragg reflection and the low $-Q_{z}$ interference fringes, at all four coverages. The fits yield a surface-normal compressive strain of $1 \%$, for all $\Theta$ 's. Thus a coverage-independent $2 \%$ vacancy concentration is incorporated in the $\mathrm{Ag} / \mathrm{Ag}(111)$ films deposited at $T=100 \mathrm{~K}$, which is the same value that was found for the (001) orientation. The other parameters obtained from the fit are given in Table I. It should be noted that the coverage computed from the fit, $\Theta^{\text {comp }}=\Sigma_{j} j P_{j}$, is in excellent agreement with the experimentally determined coverage $\Theta^{\exp }$ obtained from a calibrated quartz microbalance. As expected, $N_{\text {pyr }}$ is observed to increase linearly with the coverage, whereas $\theta_{\text {top }}$ is found to decrease due to the narrowing of the pyramid tops as they grow taller. In addition, our analysis reveals details of the surface morphology that produce the low- $Q_{z}$ interference fringes. Figure 7 shows the fraction of the exposed surface atoms, $P_{j}$, as a function of their height level $j$ (normalized to the average pyramid height $N_{\text {pyr }}$ ) at different coverages, as it results from the fits in Fig. 4. In addition to a maximum population of exposed terraces occurring for $j=N_{\text {pyr }}$, significant populations of exposed terraces also occur at small $j$. It is this extended distribution of exposed terraces, and not the mere presence of pyramids, which leads to the appearance of fringes in the low- $Q_{z}$ reflectivity. For comparison, the inset to Fig. 7 shows the " $P_{j}$ vs $j$ " dependence determined for $\mathrm{Ag} / \mathrm{Ag}(111)$ at $200 \mathrm{~K}$, [from Fig. 1(a)], where mounds are well developed, ${ }^{12}$ but no interference fringes are observed.

There are a number of interesting observations about the surface. First, it is much rougher than at $200 \mathrm{~K}$, exhibiting a more vertical growth that exposes terraces near the 


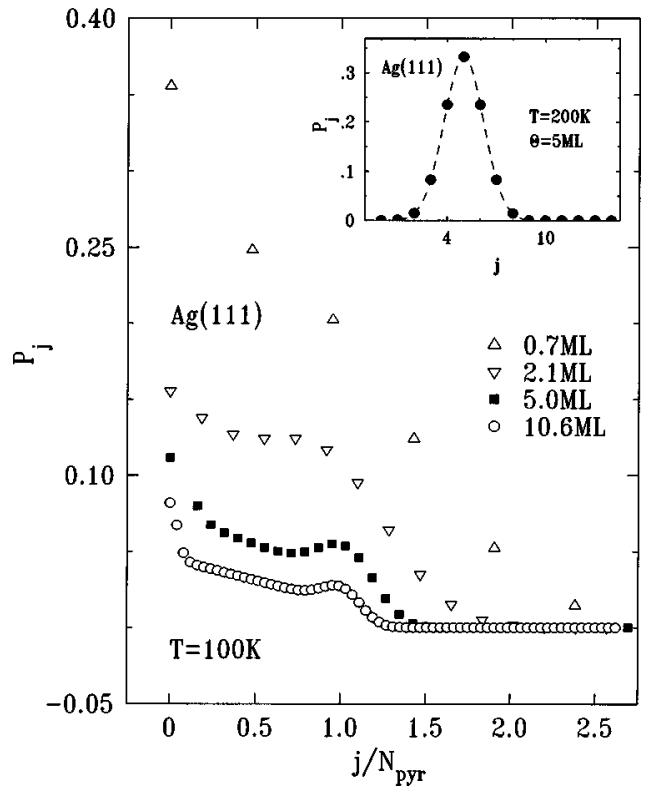

FIG. 7. Fraction of exposed surface atoms $P_{j}$, resulting from the best fits to the $\operatorname{Ag}(111)$ reflectivity data in Fig. 4, is shown as a function of the normalized height level $N_{\text {pyr }}$ for four different coverages. These particular $P_{j}$ distributions (and not the mere presence of mounds) give rise to the interference fringes observed in the low-angle region in our experiment. The inset shows the " $P_{j}$ vs $j$ " dependence for a $\mathrm{Ag} / \mathrm{Ag}(111)$ film grown at $T=200 \mathrm{~K}$ having a Gaussian distribution, which does not lead to interference fringes.

substrate (at least for these low coverages). Also the total mean-squared roughness computed from the fits, $\sigma$ $=\sqrt{\sum_{j} j^{2} P_{j}-\Theta^{2}}$, increases almost linearly with the coverage $\left(\sigma \propto \Theta^{\beta}\right.$, where $\left.\beta=1\right)$, as shown in Fig. 8, while the fluctuation of the pyramid heights is comparatively small. $\beta=1$ is what one would expect for a surface roughness dominated by geometric structures rather than noise. Finally, we observe that warming the sample to $300 \mathrm{~K}$ causes the fringes to disappear, indicating that the surface morphology changes when the vacancies anneal. This suggests that there is a large atomic mobility when the vacancies anneal, causing these rather unstable vertical structures to rapidly deteriorate through a downhill atom current, which fills the lower terraces near the substrate and destroys the interference fringes. In addition to the usual surface-curvature-driven smoothening, ${ }^{28}$ cascade or avalanche effects ${ }^{29}$ observed on both $\mathrm{Cu}(111)$ and $\mathrm{Ag}(111)$ at higher temperatures might dramatically enhance the surface annealing concomitantly with the vacancies annealing to the surface.

Thus, significant changes in the morphology of the evolving surface occur when the growth of $\mathrm{Ag}$ films on $\mathrm{Ag}(111)$ is carried out at "very" low temperatures. That these changes are concomitant with the incorporation of a large vacancy concentration in the growing film is unlikely to be coincidental. Yet, whether the vacancy formation influences the growth morphology via the induced strain (e.g., changes in activation barriers) or through changes in local kinetics around a

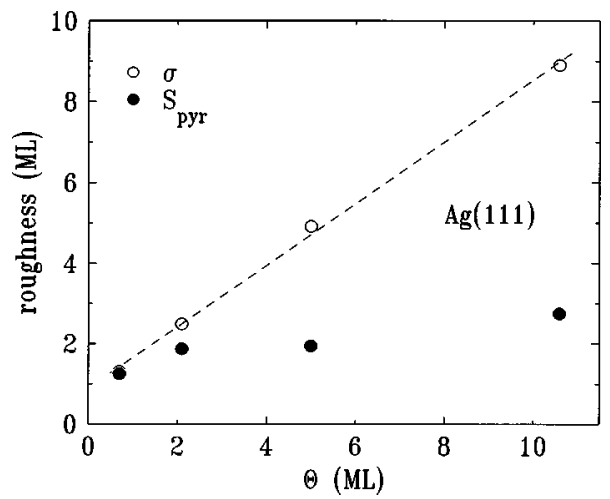

FIG. 8. Coverage dependence of the total surface roughness $\sigma$ (open symbols) and pyramid rms height fluctuations $S_{\text {pyr }}$ (solid symbols) determined from the fits to the $\mathrm{Ag}(111)$ data in Fig. 4.

vacancy or vacancy cluster is not clear, and further experimental and theoretical investigation is necessary to address these questions, as well as the full nature of the induced surface morphology.

The present study does not address the origin of the vacancies or vacancy clusters. However, the similar strain observed for both the (111) and (001) surfaces suggests that, whatever the mechanism of vacancy formation, it should be similar on both surface orientations. Candidate mechanisms include "microprotrusions," proposed by simulations, ${ }^{6}$ or possibly grain boundaries, which might precipitate vacancies or clusters. It is not clear whether either of these mechanisms would work the same way on the two surface orientations. Finally, it could be that islands trap vacancy holes during low-temperature growth and do not fill in efficiently during subsequent growth. This last mechanism has the potential to be similarly effective on both surface orientations.

In summary, we have used synchrotron x-ray diffraction to investigate the growth of $\mathrm{Ag}$ on $\mathrm{Ag}(001)$ and $\mathrm{Ag}(111)$ surfaces at $T=100 \mathrm{~K}$. We observed that a $1 \%$ compressive strain is present in the deposited Ag film, regardless of the orientation of the substrate. This indicates that a large vacancy concentration (2\%) is incorporated in the evolving film during growth. We also find that the presence of vacancies has a strong effect on the $\mathrm{Ag}(111)$ surface morphology, where the distribution of heights is much different from the Gaussian distribution found at higher temperatures.

\section{ACKNOWLEDGMENTS}

Support is acknowledged from the National Science Foundation under Contract (P.W.S.) No. DMR-9202528 and (P.F.M., C.E.B., W.C.E.) No. DMR-9623827 and the Midwest Superconductivity Consortium (MISCON) under DOE Grant No. DE-FG02-90ER45427. The SUNY X3 beam line is supported by the DOE, under Contract No. DE-FG0286ER45231, and the NSLS is supported by the DOE, Division of Material Sciences and Division of Chemical Sciences. 
${ }^{1}$ H.-J. Ernst, F. Fabre, and J. Lapujoulade, Surf. Sci. Lett. 275, L682 (1992); H.-J. Ernst, F. Fabre, R. Folkerts, and J. Lapujoulade, Phys. Rev. Lett. 72, 112 (1994).

${ }^{2}$ R. Kunkel, B. Poelsema, L. K. Verheij, and G. Comsa, Phys. Rev. Lett. 65, 733 (1990).

${ }^{3}$ J. W. Evans, D. E. Sanders, P. A. Thiel, and A. E. DePristo, Phys. Rev. B 41, 5410 (1990).

${ }^{4}$ M. C. Bartelt and J. W. Evans, Phys. Rev. Lett. 75, 4250 (1995).

${ }^{5}$ J. G. Amar and F. Family, Phys. Rev. B 54, 14742 (1996).

${ }^{6}$ C. R. Stoldt, K. J. Caspersen, M. C. Bartelt, C. J. Jenks, and J. W. Evans, Phys. Rev. Lett. 85, 800 (2000); K. J. Caspersen, C. R. Stoldt, A. R. Layson, M. C. Bartelt, P. A. Thiel, and J. W. Evans, Phys. Rev. B 63, 085401 (2001).

${ }^{7}$ C. L. Kelchner and A. E. DePristo, Surf. Sci. 393, 72 (1997).

${ }^{8}$ I. K. Robinson and D. J. Tweet, Rep. Prog. Phys. 55, 559 (1992).

${ }^{9}$ R. Feidenhans'1, Surf. Sci. Rep. 10, 105 (1989).

${ }^{10}$ I. K. Robinson, E. Vlieg, H. Hornis, and E. H. Conrad, Phys. Rev. Lett. 67, 1890 (1991); E. Vlieg, A. W. Denier van der Gon, J. F. van der Veen, J. E. Macdonald, and C. Norris, ibid. 61, 2241 (1988).

${ }^{11}$ G. Helgesen, D. Gibbs, A. P. Baddorf, D. M. Zehner, and S. G. J. Mochrie, Phys. Rev. B 48, 15320 (1993); C. E. Botez, W. C. Elliott, P. F. Miceli, and P. W. Stephens, ibid. 63, 113404 (2001).

${ }^{12}$ W. C. Elliott, P. F. Miceli, T. Tse, and P. W. Stephens, Physica B 221, 65 (1996).

${ }^{13}$ W. C. Elliott, P. F. Miceli, T. Tse, and P. W. Stephens, Phys. Rev. B 54, 17938 (1996); W. C. Elliott, P. F. Miceli, T. Tse, and P. W. Stephens, in Surface Diffusion: Atomistic and Collective Processes, Vol. 360 of NATO Advanced Study Institute, Series B: Physics, edited by M. C. Tringides (Plenum, New York, 1997), p. 209.

${ }^{14}$ P. T. Sprunger and E. W. Plummer, Phys. Rev. B 48, 14436 (1993).
${ }^{15}$ S. A. de Vries, W. J. Huisman, P. Goedtkindt, M. J. Zwanenburg, S. L. Bennett, and E. Vlieg, Phys. Rev. Lett. 81, 381 (1998).

${ }^{16}$ J. Camarero, J. de la Figuera, J. J. de Miguel, R. Miranda, J. Alvarez, and S. Ferrer, Surf. Sci. 459, 191 (2000).

${ }^{17}$ W. H. Zachariasen, Theory of X-ray Diffraction in Crystals (Dover, New York, 1967).

${ }^{18}$ R. W. Balluffi, J. Nucl. Mater. 69-70, 240 (1978).

${ }^{19}$ I. K. Robinson, W. Moritz, F. Jona, and M. A. Van Hove, Phys. Rev. Lett. 76, 3659 (1996).

${ }^{20}$ P. F. Miceli, C. J. Palmstrøm, and K. W. Moyers, Appl. Phys. Lett. 58, 1602 (1991).

${ }^{21}$ J. D. Eshelby, J. Appl. Phys. 25, 255 (1954).

${ }^{22}$ L. D. Landau and E. M. Lifshitz, Theory of Elasticity (Pergamon, New York, 1970).

${ }^{23}$ C. W. Tucker and J. B. Sampson, Acta Metall. 2, 433 (1954); T. Broom and R. K. Ham, Vacancies and Other Point Defects in Metals and Alloys, Institute of Metals Monograph and Report Series No. 23 (Institute of Metals, London, 1958), p. 41.

${ }^{24}$ M. Kalff, P. Smilauer, G. Comsa, and T. Michely, Surf. Sci. 426, L447 (1999).

${ }^{25}$ J. Villain, J. Phys. I 1, 19 (1991); G. Ehrlich and F. G. Hudda, J. Chem. Phys. 44, 1039 (1966); R. L. Schwoebel and E. J. Shipsey, J. Appl. Phys. 37, 3682 (1966).

${ }^{26}$ J. Vrijmoeth, H. A. van der Vegt, J. A. Meyer, E. Vlieg, and R. J. Behm, Phys. Rev. Lett. 72, 3843 (1994); K. Bromann, H. Brune, H. Roder, and K. Kern, ibid. 75, 677 (1995).

${ }^{27}$ P. F. Miceli, in Semiconductor Interfaces, Microstructures and Devices: Properties and Applications, edited by Z. C. Feng (IOP, Bristol, 1993), p. 87.

${ }^{28}$ W. W. Mullins, J. Appl. Phys. 28, 333 (1957).

${ }^{29}$ M. Giesen, G. Schultze Icking-Konert, and H. Ibach, Phys. Rev. Lett. 80, 552 (1998). 